\title{
Treatment of Intra-Abdominal Hydatid Cyst Using Modified PAIR Technique: Single Center Experiences
}

\author{
Enes Duman, ${ }^{1,}{ }^{*}$ Egemen Cifci, ${ }^{1}$ and Erkan Yildirim ${ }^{1}$ \\ ${ }^{1}$ Department of Interventional Radiology, Konya Research Center, Baskent University Medical Faculty, Konya, Turkey \\ "Corresponding author: Enes Duman, Department of Interventional Radiology, Konya Research Center, Baskent University Medical Faculty, Saray cad. Hocacihan mah. 42080 \\ Selcuklu, Konya, Turkey. Tel: +90-3322570606/1912, Fax: +90-332 2476886, E-mail: drenesduman@hotmail.com
}

Received 2016 May 09; Revised 2016 December 22; Accepted 2017 February 24.

\begin{abstract}
Background: Percutaneous treatment of the hydatid cyst has been well documented that morbidity and mortality rates, hospitalization time, and recurrence rate associated with percutaneous treatment are significantly less than those with surgery.

Objectives: To research the success rate of treatment of hydatid cyst by modified percutaneous treatment using a trocar technique. Patients and Methods: Patients who had intra-abdominal hydatid cyst were treated by a modified percutaneous technique were included the study. This study included patients who were treated between June- 2003 and April-2015. We excluded patients who either had biliary fistula during the intervention or did not participate in the follow-up sessions. Medical treatment with albendazole was performed before and after the procedure. Patients were followed up at 3, 9, 12, 24, 36, and 48 months after the procedure.

Results: Out of a total 83 patients, 10 patients had biliary fistula, so they underwent surgical treatment. 13 patients did not come to the controls. As a result, 60 patients who had 72 intra-abdominal hydatid cysts underwent percutaneous ablation treatment. 48 patients who had 59 hydatid cysts were cured after the first intervention. After initial treatment, the success rate was $81,9 \%$, and after the secondary intervention, the success rate was $94,4 \%$. The average diameter of cysts that were cured in a single session was $6,9 \pm$ $2,2 \mathrm{~cm}$; the average diameter of cysts that were removed during the secondary intervention and surgical treatment was 10,2 $\pm 3,4$ $\mathrm{cm}$.

Conclusion: Whatever the hydatid cyst size, if a drainage catheter is used for initial puncture using the trocar technique, it will be safer and more comfortable for patients compared with the PAIR or catheterization technique.
\end{abstract}

Keywords: Hydatid Cyst, Percutaneous Treatment, PAIR, Trocar Technique, Drainage

\section{Background}

Cystic Echinococcosis or hydatidosis is an infestation caused by larval forms of the dog tapeworm, Echinococcus granulosus. It is endemic in the Mediterranean region, South America, Australia, New Zealand and cattle rearing areas like Turkey and South East Asia (1). Hydatid cyst can develop in any organ of the body, but commonly occurs in the liver. The right lobe of the liver is affected more frequently than the left (2). The traditional treatment method of hydatid cyst is surgery. In the 1980s, reports of accidental punctures of hydatid cysts without any complications contributed to deliberate puncture of hydatid cyst followed by introduction of a scolicidal agent (3). This method was known as puncture-aspiration-injectionreaspiration (PAIR) and was recommended by the WHO as an alternative method to surgery which has been used in many centers during the past decade, has proved effective for management of the disease. Furthermore, it has been well documented that morbidity and mortality rates, hospitalization time, and recurrence rate associated with percutaneous treatment are significantly less than those with surgery $(3,4)$. Long-term results indicate that percutaneous treatment of liver hydatid cysts is an effective treatment for type I, II and III cysts $(4,5)$.

In the standard PAIR technique or catheterization technique, an initial puncture is made with an 18-gauge needle under the guidance of sonogram or CT, where all of the fluid in the cyst is aspirated. In our technique drainage catheter is used for initial puncture.

\section{Objectives}

The purpose of this study was to evaluate the success rate of treatment of hydatid cyst by modified PAIR technique using trocar technique.

\section{Patients and Methods}

Our study was a retrospective study. We informed the patients before beginning the treatment, and they signed the patient consent form. We examined each patient's files; patients who had intra-abdominal hydatid cyst that were treated by modified percutaneous technique using 
the trocar technique between June-2003 and April-2015 were included in the study. Inclusion criteria were patients with single or multiple hydatid cysts, adequate liver parenchyma surrounding the cyst ( $\geq 5 \mathrm{~mm}$ ), and patients who refused surgery and relapsed after surgery. Exclusion criteria were cysts that were inaccessible to puncture, surrounded with inadequate liver parenchyma $(<5 \mathrm{~mm})$, patients with biliary fistula, patients with Gharbi IV-V hydatid cysts, and patients with multiseptate hydatid cysts. We registered patients' gender, ages, cysts' location, numbers, size, type, follow-up period, and complications. Patients were controlled by ultrasound (Hitachi, Tokyo, Japan) to determine cyst features. Before using albendazole (Andazol; Biofarma, Istanbul, Turkey) treatment, all blood values were determined. Albendazole (Andazol; Biofarma, Istanbul, Turkey) tablet (10 mg/kg body weight) was started 14 days prior to the procedure. If the patient had a normal international normalized ratio (INR) value and had no low platelet count, treatment was started. The patients did not eat anything all night before the procedure. We excluded the patients who had biliary fistula in the intervention and did not participate in the follow-up sessions.

All procedures were performed by an interventional radiologist using a percutaneous technique using sonography. As a premedication for hypersensitivity reactions, prednisolone $25 \mathrm{mg}$ and pheniramine maleate $50 \mathrm{mg}$ were administered intravenously 20 minutes before the procedure. The patients were positioned supine or semi-supine on a fluoroscopy table with rotating C-arm equipment. A7F drainage catheter (Argon medical devices, Athens, Canada, USA) was placed in the hydatid cyst by a single puncture under standard sterile conditions with light sedation and local anesthesia by sonographic guidance (Hitachi, Tokyo, Japan). The cyst content was aspirated and we examined color and content of the cyst fluid to rule out biliary communication. To be sure, then the cyst cavity was evaluated with fluoroscopy using nonionic contrast agent to assess possible biliary fistula. If there was no biliary fistula, hypertonic saline $(20 \% \mathrm{NaCl})$ was injected into the cyst cavity up to $30 \%-35 \%$ of the estimated cyst volume. After waiting for 15 minutes, the hypertonic saline was aspirated; then, absolute alcohol was injected into the cyst cavity up to 30$35 \%$ of the estimated cyst volume. 15 minutes later, alcohol was aspirated, and then we finished the operation by removing the catheter (Figure 1). Saline has a better effect on inactivating protoscolices than alcohol; it separates laminated and germinal membranes of hydatid cysts completely from the pericyst. Alcohol is a stronger sclerosing agent than hypertonic saline, so it was injected for sclerosing to minimize and prevent the possibility of fluid collection in long term. So we used both alcohol and hypertonic saline to treat the hydatid cysts. We did not punc- ture the cyst wall directly. We always punctured the cyst wall via passing through the liver parenchyma. If there was no distance to puncture the cyst wall by passing the liver parenchyma, we did not perform percutaneous treatment and patients underwent surgical treatment. So, the chance of late leakage and anaphylaxis was minimized.

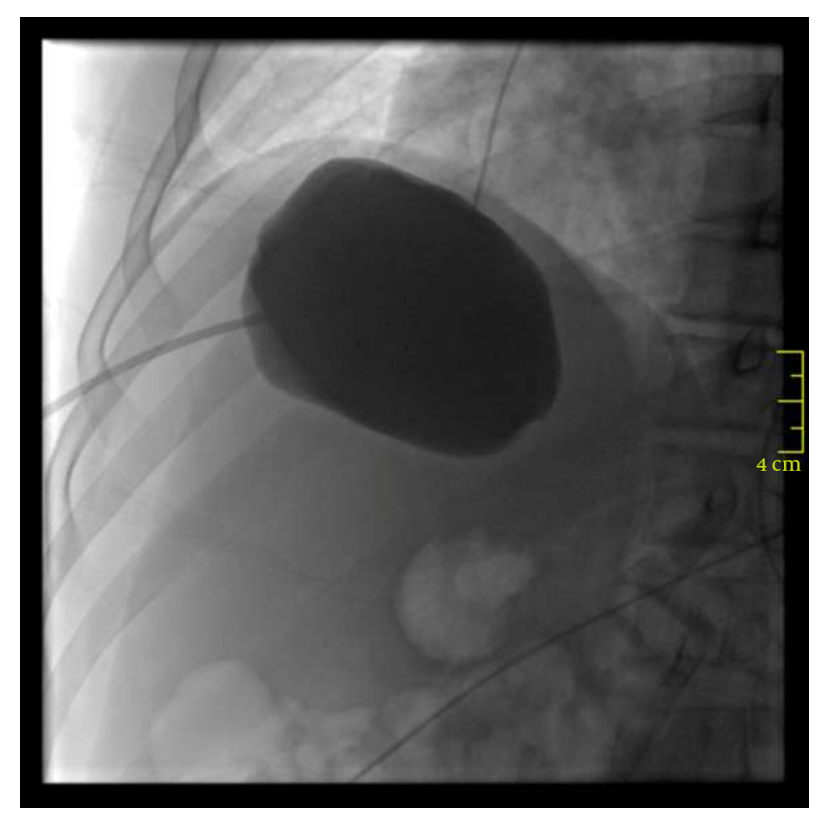

$\overline{\text { Figure 1. Contrast agent was administered through the drainage catheter and there }}$ was no extravasation.

We used modified PAIR technique for all patients because we wanted to minimize all risks of standard PAIR treatment and catheterization technique. $18 \mathrm{G}$ needle is used to puncture the cyst in the standard PAIR treatment and catheterization technique. The biggest disadvantage is that the needle tip can be plugged during aspiration of the cyst contents, and a secondary puncture may be necessary. At the same time, securing the needle in cysts is a second problem for overweight patients. While patients change their position, the needle may be removed from the cyst, and cyst contents may discharge to the peritoneum. We performed a single puncture via drainage catheter, not a needle. The catheter was soft, and its distal tip was like a pig's tail when we placed it into the cysts. Therefore, it was easier that the catheter was stable in cysts during the procedure and when patients changed positions. Plugging of the catheter was minimized because there were many holes in the tip of the catheter.

After our procedure, all patients were followed up for possible early complications in our unit. If there was no complication one or two hours later, the patients were 
discharged. Albendazole (Andazol; Biofarma, Istanbul, Turkey) was continued at the same dose for 3 months. Patients took albendazole for two weeks and then they stopped for one week. Finally, they started again after the procedure. Patients were followed up at 3, 9, 12, 24, 36, and 48 months after the procedure.

Criteria of good response were defined as reduction in the size of the cyst, changes in the echo pattern with appearance of solid areas in the cyst, progressive solidification of the cyst, calcification of the wall, and increase in echogenicity of the cyst with pseudomass appearance.

\subsection{Statistical Analyses}

All analyses were done using SPSS 9.0 (SPSS for Windows 9.0, Chicago, IL) software package. Continuous variables were presented as mean (SD), whereas categorical variables were presented as frequencies. To assess between categorical variables, the Pearson $\mathrm{X}^{2}$ test was used. In order to test whether the data were normally distributed, Kolmogorov-Smirnov test was applied. Correlations between the parameters and the association with other variables were studied using Pearson's correlation coefficient if the data met the criteria for normal distribution or using the Spearman correlation test if otherwise. A P value of $<0.05$ was considered significant.

\section{Results}

Eighty-three patients had percutaneous treatment performed for intra-abdominal hydatid cyst between 2003 and 2015. Ten patients had biliary fistula, so they underwent surgical treatment. Thirteen patients did not come to the follow-up sessions. These patients were excluded from the study. As a result, 60 patients who had 72 intraabdominal hydatid cysts underwent percutaneous ablation treatment. Fifteen patients were male (25\%), and 45 patients were female (75\%). The average age was $52 \pm 18.6$. The average diameters of hydatid cysts were $7,5 \pm 2.8$ ( $\mathrm{min}: 2.5$ - max: 18). Fifty-one cysts (70.8\%) were Gharbi type 1,19 cysts (26.4\%) were Gharbi type 2, and two patients (2.8\%) were Gharbi type 3. The average follow-up time was $42.2 \pm 16.1$ (min: 8 - max: 85) months. Fifty-seven patients had liver hydatid cyst, two patients had kidney hydatid cyst, and one patient had spleen hydatid cyst.

Three patients required secondary intervention. The average diameter of hydatid cyst in these patients was 12.1 $\pm 4.7 \mathrm{~cm}$. These patients were considered recurrence. One patient had an abscess, so we placed the drainage catheter for three weeks with medical therapy. After the control catheter was removed.
Nine hydatid cysts did not show enough improvement by sonographic guidance on controls. They underwent secondary intervention. The average diameter of hydatid cyst in these patients was $9.4 \pm 2.6 \mathrm{~cm}$. All of them were cured after secondary intervention.

Forty-eight patients ( 59 hydatid cysts) were cured after the first intervention. The average diameter of hydatid cyst in these patients was $6.9 \pm 2.2$ (min: 3 - max: 12 ) cm (Table 1). Only one patient had anaphylaxis while the intervention and medical treatment was performed. After initial treatment, the success rate was $81.9 \%$, and after the secondary intervention, the success rate was $94.4 \%$.

We compared the diameters of the cysts that were cured in a single session and the diameters of cysts that required secondary intervention and surgical treatment. The average diameter of cysts that were cured in a single session (59 cysts) was $6.9 \pm 2.2$ (min: 2.5 - max: 12 ) cm. The average diameter of cysts that required secondary intervention and surgical treatment was 10, $2 \pm 3.4$ (min: 6.5max: 18) cm. This difference was statistically significant (P $<0.001$ ). There was no statistically significant difference regarding patients' sex, age, and type of hydatid cyst between these two groups (for sex, P: 0.862; for age, P: 0.665; for type of hydatid cyst, P: 0.625). Anaphylaxis and abscess were seen in one patient $(1,7 \%)$, and allergic reactions were seen in three patients (5\%).

\section{Discussion}

PAIR therapy along with oral albendazole is very effective and has good results when compared with surgery for Gharbi type I-III cysts (6). PAIR treatment with medical therapy is a significantly reliable method to treat hydatid cyst $(6,7)$. Before and after the procedure, using albendazole decreases the possibility of recurrences (8). We also gave albendazole to all patients before and after the procedure.

The technical success of PAIR varies between $88 \%$ and $100 \%$, according to several published sources. The recurrence rate is between $1.6 \%$ and $10.9 \%$, anaphylaxia $0.03 \%$, while the mortality rate is so low it can be negated. A morbidity rate varies between $25.2 \%$ and $32 \%$, including cyst infection (22\%) and biliary fistulae (8.9\%). Length of hospitalization depends on whether a drainage catheter is used or not, and then the length of stay varies from 2.1 to 8 days, according to documented sources (9-11). Nearly 10,000 cases with hydatid cyst have been treated by the PAIR technique, and it can be concluded that PAIR is a safe and effective method for the treatment of non-complicated hydatid cysts as a first choice therapeutic modality. In our technique, the success rate was $81.9 \%$ after the first intervention; it was $94.4 \%$ after secondary intervention. The aver- 


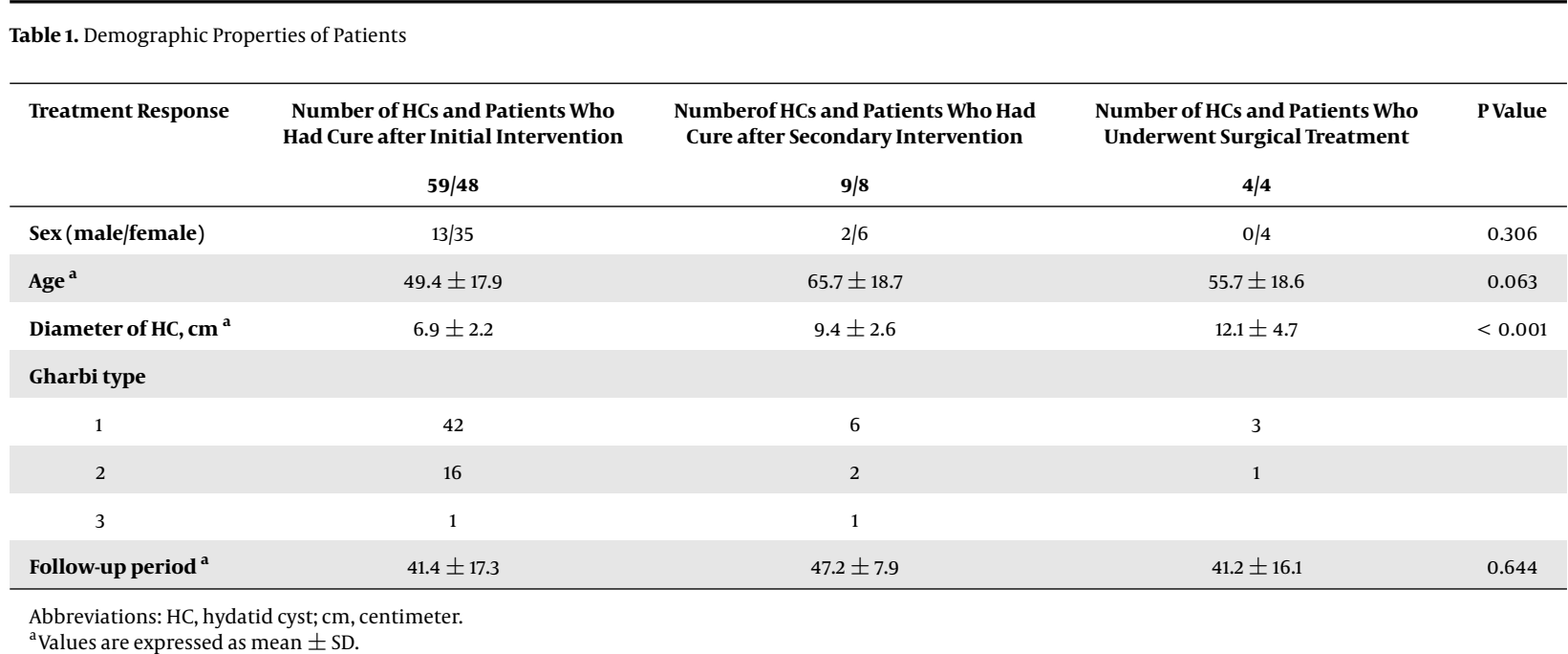

age diameter of cysts that were cured in a single session (59 cysts) was $6.9 \pm 2.2 \mathrm{~cm}$, and the average diameter of cysts that required secondary intervention and surgical treatment was $10.2 \pm 3.4 \mathrm{~cm}$. This difference was statistically significant. According to the results of our study, the percutaneous treatment method by a modified single puncture (trocar technique) can be regarded as a highly effective and reliable outpatient treatment method for hydatid cyst whose diameters are under $7.4 \mathrm{~cm}$. If hydatid cyst diameters were greater than $7.4 \mathrm{~cm}$, we could also use trocar technique, but we would not remove the catheter. One day later, we would remove the catheter to ensure that there was no need for additional processing. So, there would be no need for secondary operation.

In the standard PAIR technique, an initial puncture is made with an 18-gauge needle under the guidance of sonogram or CT, where all of the fluid in the cyst is aspirated. Scolicidal agents, generally pure alcohol (95\% concentrate) or a minimum of $20 \%$ saline, is injected into the cyst cavity, replacing $1 / 3$ of the original volume removed from the cyst. After 15 - 30 minutes, the scolocidal agents are reaspirated. In the catheterization technique, all processes are performed, then a catheter is placed in the hydatid cyst. One day later, after controlling, the catheter was removed.

An $18 \mathrm{G}$ needle is used to puncture the cyst in the standard PAIR treatment. The biggest disadvantage of this technique is that the needle tip can be plugged during aspiration of the cyst contents, and a secondary puncture may be necessary. At the same time, to secure the needle in cysts is a second problem for overweight patients. While patients change their position, the needle may be removed from the cyst, and cyst contents may discharge to the peritoneum. To minimize all these risks in our tech- nique; we used 7F drainage catheters to puncture the cysts, and we performed all operations like aspiration, ablation from that catheter. We performed a single puncture. The catheter was soft, and its distal tip was like a pig's tail when we placed it into the cysts; so, it was easier that the catheter was stable in cysts during the procedure and when patients changed positions. Plugging of the catheter was minimized because there were many holes in the tip of the catheter. If we want the catheter to remain in cysts for 24 hours, we do not need extra operation, and we can use it for free drainage to follow-up so risk of complication will decrease. For this reason, whatever the size of the hydatid cyst, we advise that the initial puncture process should be made by a $7 \mathrm{~F}$ drainage catheter by using the trocar technique, and percutaneous treatment should be made as an outpatient treatment for hydatid cysts $7.4 \mathrm{~cm}$ in size. For hydatid cysts greater than $7.4 \mathrm{~cm}$, treatment should be made by waiting for 24 hours. Nayman et al. used the trocar technique for liver hydatid cyst in their study, but they placed the catheter into the cyst for 24 hours, not outpatient (12). In our study, patients were generally treated outpatiently.

Our limitation in this study was the small number of patients that participated. Our technique should be performed in a large series.

As a conclusion, to the best of our knowledge, this is the first study confirming that hydatid cysts can be treated with minimal invasion with our technique without need for hospitalization, which is required by catheterization techniques. So, cost-effectiveness of our technique is better than the others. Our technique is also effective and safe with lower complication rates. So, every effort should be made to finish the treatment with our technique. What- 
ever the size of the hydatid cyst, if a drainage catheter is used for initial puncture, the risk of complication should be minimized. We advise that the initial puncture process should be made by a $7 \mathrm{~F}$ drainage catheter using the trocar technique, and percutaneous treatment should be made given as an outpatient treatment for hydatid cysts under $7.4 \mathrm{~cm}$ in size. For hydatid cysts greater than 7.4 $\mathrm{cm}$, treatment should be made by waiting for 24 hours with catheter, so there will be no need for extra operation. Our study emphasizes that initial puncture should be done with a drainage catheter using trocar technique.

\section{Footnote}

Conflict of Interest: All authors declared that they had no conflict of interest.

\section{References}

1. Ustunsoz B, Akhan O, Kamiloglu MA, Somuncu I, Ugurel MS, Cetiner S. Percutaneous treatment of hydatid cysts of the liver: long-term results. AJR Am J Roentgenol. 1999;172(1):91-6. doi: 10.2214/ajr.172.1.9888746. [PubMed: 9888746].

2. Nepalia S, Joshi A, Shende A, Sharma SS. Management of echinococcosis. J Assoc Physicians India. 2006;54:458-62. [PubMed: 16909694].

3. Akhan O, Ozmen MN, Dincer A, Sayek I, Gocmen A. Liver hydatid disease: long-term results of percutaneous treatment. Radiology. 1996;198(1):259-64. doi: 10.1148/radiology.198.1.8539390. [PubMed: 8539390].

4. Yagci G, Ustunsoz B, Kaymakcioglu N, Bozlar U, Gorgulu S, Simsek A, et al. Results of surgical, laparoscopic, and percutaneous treatment for hydatid disease of the liver: 10 years experience with 355 patients. World J Surg. 2005;29(12):1670-9. doi: 10.1007/s00268-0050058-1. [PubMed:16311852].

5. Polat KY, Balik AA, Oren D. Percutaneous drainage of hydatid cyst of the liver: long-term results. HРB (Oxford). 2002;4(4):163-6. doi: 10.1080/13651820260503800. [PubMed: 18332947].

6. Etlik O, Arslan H, Bay A, Sakarya ME, Harman M, Temizoz O, et al. Abdominal hydatid disease: long-term results of percutaneous treatment. Acta Radiol. 2004;45(4):383-9. [PubMed: 15323389].

7. Goktay AY, Secil M, Gulcu A, Hosgor M, Karaca I, Olguner M, et al. Percutaneous treatment of hydatid liver cysts in children as a primary treatment: long-term results. J Vasc Interv Radiol. 2005;16(6):831-9. doi: 10.1097/01.RVI.0000157777.33273.3B. [PubMed: 15947047].

8. Akhan O, Yildiz AE, Akinci D, Yildiz BD, Ciftci T. Is the adjuvant albendazole treatment really needed with PAIR in the management of liver hydatid cysts? A prospective, randomized trial with short-term follow-up results. Cardiovasc Intervent Radiol. 2014;37(6):1568-74. doi: 10.1007/s00270-014-0840-2. [PubMed: 24464258].

9. Smego RJ, Bhatti S, Khaliq AA, Beg MA. Percutaneous aspirationinjection-reaspiration drainage plus albendazole or mebendazole for hepatic cystic echinococcosis: a meta-analysis. Clin Infect Dis. 2003;37(8):1073-83. doi:10.1086/378275. [PubMed: 14523772].

10. Golemanov B, Grigorov N, Mitova R, Genov J, Vuchev D, Tamarozzi F, et al. Efficacy and safety of PAIR for cystic echinococcosis: experience on a large series of patients from Bulgaria. Am J Trop Med Hyg. 2011;84(1):48-51. doi: 10.4269/ajtmh.2011.10-0312. [PubMed: 21212200].

11. Yasawy MI, Mohammed AE, Bassam S, Karawi MA, Shariq S. Percutaneous aspiration and drainage with adjuvant medical therapy for treatment of hepatic hydatid cysts. World J Gastroenterol. 2011;17(5):646-50. doi:10.3748/wjg.v17.i5.646. [PubMed: 21350714].

12. Nayman A, Guler I, Keskin S, Erdem TB, Borazan H, Kucukapan A, et al. A novel modified PAIR technique using a trocar catheter for percutaneous treatment of liver hydatid cysts: a six-year experience. $D i$ agn Interv Radiol. 2016;22(1):47-51. doi:10.5152/dir.2015.15011. [PubMed: 26574902]. 